\title{
Boundedness of sublinear operators and their commutators on generalized central Morrey spaces
}

\section{Yun Fan*}

\section{"Correspondence:}

fanyun1522@163.com

Department of Mathematics,

Huzhou Teachers College, Huzhou,

Zhejiang 313000, P.R. China

\begin{abstract}
In this paper, we introduce the generalized central Morrey spaces $\dot{B}^{p, \varphi}\left(\mathbb{R}^{n}\right)$ and get the boundedness of a large class of rough operators on them. We also consider the $\mathrm{CBMO}$ estimates of their commutators on generalized central Morrey spaces. As applications, we obtain the boundedness characterizations of rough

Hardy-Littlewood maximal function, rough Calderón-Zygmund singular integral, rough fractional integral, etc. on generalized central Morrey spaces.
\end{abstract}

MSC: 42B20; 42B25

Keywords: sublinear operators; commutator; central Morrey spaces; rough kernel

\section{Introduction}

Let $\Omega \in L^{s}\left(S^{n-1}\right)$ be homogeneous of degree zero on $\mathbb{R}^{n}$, where $S^{n-1}$ denotes the unit sphere of $\mathbb{R}^{n}$ and $s>1$. We define $s^{\prime}=s /(s-1)$ for any $s>1$. Suppose that $T_{\Omega}$ represents a sublinear operator, which satisfies that for any $f \in L^{1}\left(\mathbb{R}^{n}\right)$ with compact support and $x \notin \operatorname{supp} f$,

$$
\left|T_{\Omega} f(x)\right| \leq C \int_{\mathbb{R}^{n}} \frac{|\Omega(x-y)|}{|x-y|^{n}}|f(y)| d y
$$

where $C>0$ is an absolute constant. Similarly, for any $0<\alpha<n$, we assume that $T_{\Omega, \alpha}$ represents a sublinear operator, which satisfies that

$$
\left|T_{\Omega, \alpha} f(x)\right| \leq C \int_{\mathbb{R}^{n}} \frac{|\Omega(x-y)|}{|x-y|^{n-\alpha}}|f(y)| d y
$$

for any $f \in L^{1}\left(\mathbb{R}^{n}\right)$ with compact support and $x \notin \operatorname{supp} f$.

Let $T$ be a linear operator. For a locally integrable function $b$ on $\mathbb{R}^{n}$, we define the commutator $[T, b]$ by

$$
[T, b] f(x)=b(x) T f(x)-T(b f)(x)
$$

for any suitable function $f$.

To study the local behavior of solutions to second-order elliptic partial differential equations, Morrey [1] introduced the classical Morrey spaces $L^{p, \lambda}\left(\mathbb{R}^{n}\right)$. The readers can find more details in [2].

() 2013 Fan; licensee Springer. This is an Open Access article distributed under the terms of the Creative Commons Attribution License (http://creativecommons.org/licenses/by/2.0), which permits unrestricted use, distribution, and reproduction in any medium, provided the original work is properly cited. 
Let $1 \leq p<\infty$ and $\lambda \geq 0 . B\left(x_{0}, t\right)$ denotes a ball centered at $x_{0}$ of radius $t$. Morrey spaces $L^{p, \lambda}\left(\mathbb{R}^{n}\right)$ are defined by

$$
L^{p, \lambda}\left(\mathbb{R}^{n}\right)=\left\{f \in L_{\mathrm{loc}}^{p}\left(\mathbb{R}^{n}\right):\|f\|_{L^{p, \lambda}\left(\mathbb{R}^{n)}\right.}<\infty\right\}
$$

where

$$
\|f\|_{L^{p, \lambda}\left(\mathbb{R}^{n}\right)}=\sup _{x_{0} \in \mathbb{R}^{n}, t>0}\left(\frac{1}{t^{\lambda}} \int_{B\left(x_{0}, t\right)}|f(x)|^{p} d x\right)^{\frac{1}{p}} .
$$

When $1 \leq p<\infty, L^{p, 0}\left(\mathbb{R}^{n}\right)=L^{p}\left(\mathbb{R}^{n}\right)$ and $L^{p, n}\left(\mathbb{R}^{n}\right)=L^{\infty}\left(\mathbb{R}^{n}\right)$; when $\lambda>n, L^{p, \lambda}\left(\mathbb{R}^{n}\right)=\{0\}$.

Many authors have studied the mapping properties of many operators on Morrey spaces; see [3-5] and [6]. Alvarez et al. [7], in order to study the relationship between central $B M O$ spaces and Morrey spaces, introduced $\lambda$-central bounded mean oscillation spaces and central Morrey spaces.

Let $\lambda<\frac{1}{n}$ and $1<p<\infty$. A function $f \in L_{\mathrm{loc}}^{p}\left(\mathbb{R}^{n}\right)$ belongs to the $\lambda$-central bounded mean oscillation spaces $C B M O^{p, \lambda}\left(\mathbb{R}^{n}\right)$ if

$$
\|f\|_{C B M O^{p}, \lambda}=\sup _{r>0}\left(\frac{1}{|B(0, r)|^{1+\lambda p}} \int_{B(0, r)}\left|f(x)-f_{B(0, r)}\right|^{p}\right)^{\frac{1}{p}}<\infty,
$$

where $f_{B(0, r)}=\frac{1}{|B(0, r)|} \int_{B(0, r)} f(y) d y$. If two functions which differ by a constant are regarded as functions in the spaces $C B M O^{p, \lambda}\left(\mathbb{R}^{n}\right)$, then $C B M O^{p, \lambda}\left(\mathbb{R}^{n}\right)$ spaces become Banach spaces. $C B M O^{p, \lambda}\left(\mathbb{R}^{n}\right)$ spaces become the spaces of constants when $\lambda<-\frac{1}{p}$ and they coincide with $L^{p}\left(\mathbb{R}^{n}\right)$ modulo constants when $\lambda=-\frac{1}{p}$.

Let $\lambda \in \mathbb{R}$ and $1<p<\infty$. The central Morrey spaces $\dot{B}^{p, \lambda}\left(\mathbb{R}^{n}\right)$ are defined by

$$
\|f\|_{B^{p}, \lambda}=\sup _{r>0}\left(\frac{1}{|B(0, r)|^{1+\lambda p}} \int_{B(0, r)}|f(x)|^{p}\right)^{\frac{1}{p}}<\infty .
$$

It follows that $\dot{B}^{p, \lambda}\left(\mathbb{R}^{n}\right)$ spaces are Banach spaces continuously included in $C B M O^{p, \lambda}\left(\mathbb{R}^{n}\right)$ spaces. $\dot{B}^{p, \lambda}\left(\mathbb{R}^{n}\right)$ spaces reduce to $\{0\}$ when $\lambda<-\frac{1}{p}$, and it is true that $\dot{B}^{p,-\frac{1}{p}}\left(\mathbb{R}^{n}\right)=L^{p}\left(\mathbb{R}^{n}\right)$, $\dot{B}^{p, 0}\left(\mathbb{R}^{n}\right)=\dot{B}^{p}\left(\mathbb{R}^{n}\right)$.

Recently, Guliyev [8] introduced the generalized Morrey spaces $L^{p, \varphi}\left(\mathbb{R}^{n}\right)$, where $\varphi(x, r)$ is a positive measurable function on $\mathbb{R}^{n} \times(0, \infty)$ and $1 \leq p<\infty$. For all functions $f \in L_{\text {loc }}^{p}\left(\mathbb{R}^{n}\right)$, the generalized Morrey spaces $L^{p, \varphi}\left(\mathbb{R}^{n}\right)$ are defined by

$$
\|f\|_{L^{p, \varphi}\left(\mathbb{R}^{n}\right)}=\sup _{x \in \mathbb{R}^{n}, r>0} \varphi(x, r)^{-1}|B(x, r)|^{-\frac{1}{p}}\|f\|_{L^{p}(B(x, r))}<\infty .
$$

Obviously, if $\varphi(x, r)=r^{\frac{\lambda-n}{p}}, L^{p, \lambda}\left(\mathbb{R}^{n}\right)=L^{p, \varphi}\left(\mathbb{R}^{n}\right)$.

When $\Omega \equiv 1$, Guliyev obtained the sufficient condition on $\varphi_{1}$ and $\varphi_{2}$

$$
\int_{r}^{\infty} \frac{\operatorname{essinf}_{t<\tau<\infty} \varphi_{1}(\tau) \tau^{\frac{n}{p}}}{t^{\frac{n}{p}+1}} d t \leq C \varphi_{2}(r)
$$


for the boundedness of $T_{\Omega}$ satisfying (1.1) from $L^{p, \varphi_{1}}\left(\mathbb{R}^{n}\right)$ to $L^{p, \varphi_{2}}\left(\mathbb{R}^{n}\right)$ in [9] and gave the condition on the pair of $\left(\varphi_{1}, \varphi_{2}\right)$

$$
\int_{r}^{\infty} \frac{\operatorname{essinf}_{t<\tau<\infty} \varphi_{1}(\tau) \tau^{\frac{n}{p}}}{t^{\frac{n}{q}+1}} d t \leq C \varphi_{2}(r)
$$

for the boundedness of $T_{\Omega, \alpha}$ satisfying (1.2) from $L^{p, \varphi_{1}}\left(\mathbb{R}^{n}\right)$ to $L^{q, \varphi_{2}}\left(\mathbb{R}^{n}\right)$ in [10], where $\frac{1}{q}=$ $\frac{1}{p}-\frac{\alpha}{n}$.

Inspired by the above, we consider the boundedness of sublinear operators on the following generalized central Morrey spaces and give the $\lambda$-central bounded mean oscillation estimates for linear operator commutators.

Definition 1.1 Let $\varphi(r)$ be a positive measurable function on $\mathbb{R}_{+}$and $1<p<\infty$. We denote by $\dot{B}^{p, \varphi}\left(\mathbb{R}^{n}\right)$ the generalized central Morrey spaces, the spaces of all $f \in L_{\mathrm{loc}}^{p}\left(\mathbb{R}^{n}\right)$ with finite quasinorm

$$
\|f\|_{\dot{B}^{p, \varphi}\left(\mathbb{R}^{n}\right)}=\sup _{r>0} \varphi(r)^{-1}|B(0, r)|^{-\frac{1}{p}}\|f\|_{L^{p}(B(0, r))} .
$$

We can recover the spaces $\dot{B}^{p, \lambda}\left(\mathbb{R}^{n}\right)$ under the choice $\varphi(r)=r^{n \lambda}$.

Recall that in 1994 the doctoral thesis [11] by Guliyev (see also [12-15]) introduced the local Morrey-type space $L M_{p \theta, \omega}$ given by

$$
\|f\|_{L M_{p \theta, \omega}}=\|\omega(r)\| f\left\|_{L^{p}(B(0, r))}\right\|_{L^{\theta}(0, \infty)}<\infty
$$

where $\omega$ is a positive measurable function defined on $(0, \infty)$. The main purpose of [11] (also of [12-15]) is to give some sufficient conditions for the boundedness of fractional integral operators and singular integral operators defined on homogeneous Lie groups in the local Morrey-type space $L M_{p \theta, \omega}$. In a series of papers by Burenkov, H Guliyev and V Guliyev, etc. (see [16-21]), some necessary and sufficient conditions for the boundedness of fractional maximal operators, fractional integral operators and singular integral operators in local Morrey-type spaces $L M_{p \theta, \omega}$ were given.

Particularly, if $\theta=\infty, L M_{p \theta, \omega}=L M_{p, \omega}$, then the generalized central Morrey spaces $\dot{B}^{p, \varphi}\left(\mathbb{R}^{n}\right)$ are the same spaces as the local Morrey spaces $L M_{p, \omega}$ with $\omega(r)=\varphi(r)^{-1} r^{-\frac{n}{p}}$.

The following statements were proved in [11] (see also [14]).

Theorem A Let $1<p<\infty$ and $\left(\varphi_{1}, \varphi_{2}\right)$ satisfy the condition

$$
\int_{r}^{\infty} \varphi_{1}(t) \frac{d t}{t} \leq C \varphi_{2}(r)
$$

where $C$ does not depend on $r$. Then the Calderón-Zygmund operator $T$ is bounded from $\dot{B}^{p, \varphi_{1}}$ to $\dot{B}^{p, \varphi_{2}}$

Theorem B Let $1<p<\infty, 0<\alpha<\frac{n}{p}, \frac{1}{q}=\frac{1}{p}-\frac{\alpha}{n}$ and $\left(\varphi_{1}, \varphi_{2}\right)$ satisfy the condition

$$
\int_{r}^{\infty} t^{\alpha} \varphi_{1}(t) \frac{d t}{t} \leq C \varphi_{2}(r)
$$

where $C$ does not depend on $r$. Then the Riesz potential $I_{\alpha}$ is bounded from $\dot{B}^{p, \varphi_{1}}$ to $\dot{B}^{q, \varphi_{2}}$. 
From Lemmas 4.4 and 5.3 in [9] we get the following for the generalized central (local) Morrey spaces $\dot{B}^{p, \varphi}$.

Theorem C Let $1<p<\infty, T$ be a sublinear operator satisfying that for any $f \in L^{1}\left(\mathbb{R}^{n}\right)$ with compact support and $x \notin \operatorname{supp} f$,

$$
|T f(x)| \leq C \int_{\mathbb{R}^{n}} \frac{|f(y)|}{|x-y|^{n}} d y,
$$

and bounded on $f \in L^{p}\left(\mathbb{R}^{n}\right)$. Let also the pair $\left(\varphi_{1}, \varphi_{2}\right)$ satisfy the condition

$$
\int_{r}^{\infty} \frac{\operatorname{essinf}_{t<\tau<\infty} \varphi_{1}(\tau) \tau^{\frac{n}{p}}}{t^{\frac{n}{p}+1}} d t \leq C \varphi_{2}(r),
$$

where $C$ does not depend on $r$. Then the operator $T$ is bounded from $\dot{B}^{p, \varphi_{1}}$ to $\dot{B}^{p, \varphi_{2}}$.

Theorem $\mathrm{D}$ Let $1<p<\infty, 0<\alpha<\frac{n}{p}, \frac{1}{q}=\frac{1}{p}-\frac{\alpha}{n}, T_{\alpha}$ be a sublinear operator satisfying that for any $f \in L^{1}\left(\mathbb{R}^{n}\right)$ with compact support and $x \notin \operatorname{supp} f$,

$$
\left|T_{\alpha} f(x)\right| \leq C \int_{\mathbb{R}^{n}} \frac{|f(y)|}{|x-y|^{n-\alpha}} d y
$$

and bounded from $f \in L^{p}\left(\mathbb{R}^{n}\right)$ to $f \in L^{q}\left(\mathbb{R}^{n}\right)$. Let also the pair $\left(\varphi_{1}, \varphi_{2}\right)$ satisfy the condition

$$
\int_{r}^{\infty} \frac{\operatorname{essinf}_{t<\tau<\infty} \varphi_{1}(\tau) \tau^{\frac{n}{p}}}{t^{\frac{n}{q}+1}} d t \leq C \varphi_{2}(r)
$$

where $C$ does not depend on $r$. Then the operator $T_{\alpha}$ is bounded from $\dot{B}^{p, \varphi_{1}}$ to $\dot{B}^{q, \varphi_{2}}$.

\section{Sublinear operator with rough kernel}

Theorem $\mathbf{E}$ Let $\omega$ be a positive weight function on $(0, \infty)$. The inequality

$$
\text { ess } \sup _{t>0} v_{2}(t) H_{\omega} g(t) \leq c \text { ess } \sup _{t>0} v_{1}(t) g(t)
$$

holds for all non-negative and non-increasing $g$ on $(0, \infty)$ if and only if

$$
A:=\sup _{t>0} \frac{\nu_{2}(t)}{t} \int_{0}^{t} \frac{\omega(r) d r}{\operatorname{ess} \sup _{0<\tau<r} \nu_{1}(\tau)}<\infty,
$$

and $c \approx A$, where the $H_{\omega}$ is the weighted Hardy operator

$$
H_{\omega} g(t):=\frac{1}{t} \int_{0}^{t} g(r) \omega(r) d r, \quad 0<t<\infty .
$$

Note that Theorem E can be proved analogously to Theorem 1 in [22]; particularly, when $\omega \equiv 1$, it was proved in [23].

In this section we are going to discuss the boundedness of $T_{\Omega}$ and $T_{\Omega, \alpha}$ on generalized central Morrey spaces.

Lemma 2.1 Let $1<p<\infty, T_{\Omega}$ be a sublinear operator and satisfy $(1.1)$ with $\Omega \in L^{s}\left(S^{n-1}\right)$. 
When $s^{\prime} \leq p$ and $T_{\Omega}$ is bounded on $L^{p}\left(\mathbb{R}^{n}\right)$ for $1<p<\infty$, then the inequality

$$
\left\|T_{\Omega} f\right\|_{L^{p}(B(0, r))} \leq C r^{\frac{n}{p}} \int_{2 r}^{\infty} t^{-\frac{n}{p}-1}\|f\|_{L^{p}(B(0, t))} d t
$$

holds for any ball $B(0, r)$ and for all $f \in L_{\mathrm{loc}}^{p}\left(\mathbb{R}^{n}\right)$; or $p<s$ and $T_{\Omega}$ is bounded on $L^{p}\left(\mathbb{R}^{n}\right)$ for $1<p<\infty$, then the inequality

$$
\left\|T_{\Omega} f\right\|_{L^{p}(B(0, r))} \leq C r^{\frac{n}{p}-\frac{n}{s}} \int_{2 r}^{\infty} t^{-\frac{n}{p}+\frac{n}{s}-1}\|f\|_{L^{p}(B(0, t))} d t
$$

holds for any ball $B(0, r)$ and for all $f \in L_{\mathrm{loc}}^{p}\left(\mathbb{R}^{n}\right)$.

Proof Let $1<p<\infty$. For any $r>0$, set $B=B(0, r)$ and $2 B=B(0,2 r)$. We write

$$
f(x)=f(x) \chi_{2 B}(x)+f(x) \chi_{(2 B)}(x):=f_{1}(x)+f_{2}(x)
$$

and have

$$
\left\|T_{\Omega_{2}} f\right\|_{L^{p}(B)} \leq\left\|T_{\Omega_{2}} f_{1}\right\|_{L^{p}(B)}+\left\|T_{\Omega_{2}} f_{2}\right\|_{L^{p}(B)} .
$$

Since $T_{\Omega} f_{1}$ is bounded on $L^{p}\left(\mathbb{R}^{n}\right)$, it follows that

$$
\left\|T_{\Omega} f_{1}\right\|_{L^{p}(B)} \leq\left\|T_{\Omega} f_{1}\right\|_{L^{p}\left(\mathbb{R}^{n}\right)} \leq C\left\|f_{1}\right\|_{L^{p}\left(\mathbb{R}^{n}\right)}=C\|f\|_{L^{p}(2 B)},
$$

where the constant $C>0$ is independent of $f$.

It is known that $x \in B, y \in(2 B)^{c}$, which implies $\frac{1}{2}|y| \leq|x-y| \leq \frac{3}{2}|y|$. Thus

$$
\left|T_{\Omega} f_{2}(x)\right| \leq C \int_{(2 B)^{c}}|f(y)||\Omega(x-y)| \frac{d y}{|y|^{n}} .
$$

(i) When $s^{\prime} \leq p$ and by Fubini's theorem, we have

$$
\begin{aligned}
& \int_{(2 B)^{c}}|f(y)||\Omega(x-y)| \frac{d y}{|y|^{n}} \\
& \quad=C \int_{(2 B)^{c}}|f(y)||\Omega(x-y)| \int_{|y|}^{\infty} \frac{d t}{t^{n+1}} d y \\
& \quad \leq C \int_{2 r}^{\infty} \int_{2 r \leq|y|<t}|f(y)||\Omega(x-y)| d y \frac{d t}{t^{n+1}} \\
& \leq C \int_{2 r}^{\infty}\|f\|_{L^{p}(B(0, t))}\left(\int_{B(0, t)}|\Omega(x-y)|^{s} d y\right)^{\frac{1}{s}}|B(0, t)|^{1-\frac{1}{p}-\frac{1}{s}} \frac{d t}{t^{n+1}} \\
& \leq C \int_{2 r}^{\infty}\|f\|_{L^{p}(B(0, t))} \frac{d t}{t^{\frac{n}{p}+1}} .
\end{aligned}
$$

Hence, for all $p \in(0, \infty)$, the inequality

$$
\left\|T_{\Omega} f_{2}\right\|_{L^{p}(B)} \leq C r^{\frac{n}{p}} \int_{2 r}^{\infty}\|f\|_{L^{p}(B(0, t))} \frac{d t}{t^{\frac{n}{p}+1}}
$$

holds. 
(ii) When $p<s$, by Fubini's theorem and the Minkowski inequality, we get

$$
\begin{aligned}
\left\|T_{\Omega} f_{2}\right\|_{L^{p}(B)} & \leq\left(\int_{B}\left|\int_{2 r}^{\infty} \int_{B(0, t)}\right| f(y)|| \Omega(x-y)\left|d y \frac{d t}{t^{n+1}}\right|^{p} d x\right)^{\frac{1}{p}} \\
& \leq \int_{2 r}^{\infty} \int_{B(0, t)}|f(y)|\left(\int_{B}|\Omega(x-y)|^{p} d x\right)^{\frac{1}{p}} d y \frac{d t}{t^{n+1}} \\
& \leq C \int_{2 r}^{\infty} \int_{B(0, t)}|f(y)|\left(\int_{B(0, t)}|\Omega(x-y)|^{s} d x\right)^{\frac{1}{s}}|B|^{\frac{1}{p}-\frac{1}{s}} d y \frac{d t}{t^{n+1}} \\
& \leq C r^{\frac{n}{p}-\frac{n}{s}} \int_{2 r}^{\infty} \int_{B(0, t)}|f(y)| d y \frac{d t}{t^{n-\frac{n}{s}+1}} \\
& \leq C r^{\frac{n}{p}-\frac{n}{s}} \int_{2 r}^{\infty}\|f\|_{L^{p}(B(0, t))} \frac{d t}{t^{\frac{n}{p}-\frac{n}{s}+1}} .
\end{aligned}
$$

On the other hand, for any $q>0$, we have

$$
\|f\|_{L^{p}(2 B)}=C r^{\frac{n}{q}}\|f\|_{L^{p}(2 B)} \int_{2 r}^{\infty} \frac{d t}{t^{\frac{n}{q}+1}} \leq C r^{\frac{n}{q}} \int_{2 r}^{\infty}\|f\|_{L^{p}(B(0, t))} \frac{d t}{t^{\frac{n}{q}+1}} .
$$

Combining the above estimates, we complete the proof of Lemma 2.1.

Theorem 2.2 Let $1<p<\infty$ and $\Omega \in L^{s}\left(S^{n-1}\right)$. Let $T_{\Omega}$ be a sublinear operator satisfying (1.1) and bounded on $L^{p}\left(\mathbb{R}^{n}\right)$ for $p>1$. If either of the two conditions

(i) when $s^{\prime} \leq p,\left(\varphi_{1}, \varphi_{2}\right)$ satisfies the condition

$$
\int_{r}^{\infty} \frac{\operatorname{essinf}_{t<\tau<\infty} \varphi_{1}(\tau) \tau^{\frac{n}{p}}}{t^{\frac{n}{p}+1}} d t \leq C \varphi_{2}(r),
$$

(ii) when $p<s,\left(\varphi_{1}, \varphi_{2}\right)$ satisfies the condition

$$
\int_{r}^{\infty} \frac{\operatorname{essinf}_{t<\tau<\infty} \varphi_{1}(\tau) \tau^{\frac{n}{p}}}{t^{\frac{n}{p}-\frac{n}{s}+1}} d t \leq C \varphi_{2}(r) r^{\frac{n}{s}}
$$

is satisfied, then the operator $T_{\Omega}$ is bounded from $\dot{B}^{p, \varphi_{1}}$ to $\dot{B}^{p, \varphi_{2}}$.

Proof When $s^{\prime} \leq p$, by Lemma 2.1 and Theorem E, for $\omega \equiv 1, p>1$, we have

$$
\begin{aligned}
\left\|T_{\Omega} f\right\|_{B^{p, \varphi_{2}}} & \leq C \sup _{r>0} \varphi_{2}(r)^{-1} \int_{r}^{\infty}\|f\|_{L^{p}(B(0, t))} \frac{d t}{t^{\frac{n}{p}+1}} \\
& =C \sup _{r>0} \varphi_{2}(r)^{-1} \int_{0}^{r^{-\frac{n}{p}}}\|f\|_{L^{p}\left(B\left(0, t^{-\frac{p}{n}}\right)\right)} d t \\
& =C \sup _{r>0} \varphi_{2}\left(r^{-\frac{p}{n}}\right)^{-1} \int_{0}^{r}\|f\|_{L^{p}\left(B\left(0, t^{-\frac{p}{n}}\right)\right)} d t \\
& \leq C \sup _{r>0} \varphi_{1}\left(r^{-\frac{p}{n}}\right)^{-1} r\|f\|_{L^{p}\left(B\left(0, r^{-\frac{p}{n}}\right)\right)}=C\|f\|_{B^{p, \varphi_{1}} .} .
\end{aligned}
$$

For the case of $p<s$, we can use the same method to prove the desirable conclusion. 
The Calderón-Zygmund operator with rough kernel $\tilde{T}_{\Omega}$ has the following integral expression:

$$
\tilde{T}_{\Omega} f(x)=\int_{\mathbb{R}^{n}} K(x, y) f(y) \Omega(y) d y
$$

for any test function $f$ and $x \notin \operatorname{supp} f$. The kernel is a locally integral function defined away from the diagonal satisfying the size condition

$$
K(x, y) \leq C \frac{1}{|x-y|^{n}}
$$

for all $x, y \in \mathbb{R}^{n}$ and $x \neq y$.

$f \in L_{\text {loc }}^{1}$, the rough Hardy-Littlewood maximal function $M_{\Omega}$ is defined by

$$
M_{\Omega} f(x)=\sup _{t>0} \frac{1}{|B(x, t)|} \int_{B(x, t)}|\Omega(y)||f(y)| d y .
$$

Then we can get the following corollary.

Corollary 2.3 Let $1<p<\infty$ and $\Omega \in L^{s}\left(S^{n-1}\right)$. If either of the two conditions

(i) when $s^{\prime} \leq p,\left(\varphi_{1}, \varphi_{2}\right)$ satisfies the condition

$$
\int_{r}^{\infty} \frac{\operatorname{essinf}_{t<\tau<\infty} \varphi_{1}(\tau) \tau^{\frac{n}{p}}}{t^{\frac{n}{p}+1}} \leq C \varphi_{2}(r),
$$

(ii) when $p<s,\left(\varphi_{1}, \varphi_{2}\right)$ satisfies the condition

$$
\int_{r}^{\infty} \frac{\operatorname{essinf}_{t<\tau<\infty} \varphi_{1}(\tau) \tau^{\frac{n}{p}}}{t^{\frac{n}{p}-\frac{n}{s}+1}} \leq C \varphi_{2}(r) r^{\frac{n}{s}}
$$

is satisfied, then $M_{\Omega}$ and $\tilde{T}_{\Omega}$ are both bounded from $\dot{B}^{p, \varphi_{1}}$ to $\dot{B}^{p, \varphi_{2}}$.

In the following statements, the boundedness of $T_{\Omega, \alpha}$ satisfying (1.2) in generalized central Morrey spaces is proved.

Lemma 2.4 Let $0<\alpha<n, 1<p<\frac{n}{\alpha}$ and $\frac{1}{q}=\frac{1}{p}-\frac{\alpha}{n}, T_{\Omega, \alpha}$ be a sublinear operator and satisfy (1.2) with $\Omega \in L^{s}\left(S^{n-1}\right)$.

When $s^{\prime} \leq p$ and $T_{\Omega, \alpha}$ is bounded from $L^{p}\left(\mathbb{R}^{n}\right)$ to $L^{q}\left(\mathbb{R}^{n}\right)$, then the inequality

$$
\left\|T_{\Omega, \alpha} f\right\|_{L^{q}(B(0, r))} \leq C r^{\frac{n}{q}} \int_{2 r}^{\infty} t^{-\frac{n}{q}-1}\|f\|_{L^{p}(B(0, t))} d t
$$

holds for any ball $B(0, r)$ and for all $f \in L_{\mathrm{loc}}^{p}\left(\mathbb{R}^{n}\right)$; or $q<s$ and $T_{\Omega, \alpha}$ is bounded from $L^{p}\left(\mathbb{R}^{n}\right)$ to $L^{q}\left(\mathbb{R}^{n}\right)$, then the inequality

$$
\left\|T_{\Omega, \alpha} f\right\|_{L^{q}(B(0, r))} \leq C r^{\frac{n}{q}-\frac{n}{s}} \int_{2 r}^{\infty} t^{-\frac{n}{q}+\frac{n}{s}-1}\|f\|_{L^{p}(B(0, t))} d t
$$

holds for any ball $B(0, r)$ and for all $f \in L_{\mathrm{loc}}^{p}\left(\mathbb{R}^{n}\right)$. 
Proof Let $0<\alpha<n, 1<p<\frac{n}{\alpha}$ and $\frac{1}{q}=\frac{1}{p}-\frac{\alpha}{n}$. For any $r>0$, set $B=B(0, r)$ and $2 B=B(0,2 r)$.

We write

$$
f(x)=f(x) \chi_{2 B}(x)+f(x) \chi_{(2 B)}(x):=f_{1}(x)+f_{2}(x)
$$

and have

$$
\left\|T_{\Omega, \alpha} f\right\|_{L^{q(B)}} \leq\left\|T_{\Omega, \alpha} f_{1}\right\|_{L^{q}(B)}+\left\|T_{\Omega, \alpha} f_{2}\right\|_{L^{q}(B)} .
$$

Since $T_{\Omega, \alpha} f_{1}$ is bounded from $L^{p}\left(\mathbb{R}^{n}\right)$ to $L^{q}\left(\mathbb{R}^{n}\right)$, it follows that

$$
\left\|T_{\Omega, \alpha} f_{1}\right\|_{L^{q}(B)} \leq\left\|T_{\Omega, \alpha} f_{1}\right\|_{L^{q}\left(\mathbb{R}^{n}\right)} \leq C\left\|f_{1}\right\|_{L^{p}\left(\mathbb{R}^{n}\right)}=C\|f\|_{L^{p}(2 B)},
$$

where the constant $C>0$ is independent of $f$.

Since $x \in B, y \in(2 B)^{c}$, thus

$$
\left|T_{\Omega, \alpha} f_{2}(x)\right| \leq C \int_{(2 B) c}|f(y)||\Omega(x-y)| \frac{d y}{|y|^{n-\alpha}} .
$$

(i) When $s^{\prime} \leq p$ and by Fubini's theorem, we have

$$
\begin{aligned}
& \int_{(2 B)^{c}}|f(y)||\Omega(x-y)| \frac{d y}{|y|^{n-\alpha}} \\
& \quad=C \int_{(2 B)^{c}}|f(y)||\Omega(x-y)| \int_{|y|}^{\infty} \frac{d t}{t^{n+1-\alpha}} d y \\
& \quad \leq C \int_{2 r}^{\infty} \int_{2 r \leq|y|<t}|f(y)||\Omega(x-y)| d y \frac{d t}{t^{n+1-\alpha}} \\
& \leq C \int_{2 r}^{\infty}\|f\|_{L^{p}(B(0, t))}\left(\int_{B(0, t)}|\Omega(x-y)|^{s} d y\right)^{\frac{1}{s}}|B(0, t)|^{1-\frac{1}{p}-\frac{1}{s}} \frac{d t}{t^{n+1-\alpha}} \\
& \leq C \int_{2 r}^{\infty}\|f\|_{L^{p}(B(0, t))} \frac{d t}{t^{\frac{n}{q}+1}} .
\end{aligned}
$$

Hence, for all $p \in(0, \infty)$, the inequality

$$
\left\|T_{\Omega, \alpha} f_{2}\right\|_{L^{q}(B)} \leq C r^{\frac{n}{q}} \int_{2 r}^{\infty}\|f\|_{L^{p}(B(0, t))} \frac{d t}{t^{\frac{n}{q}+1}}
$$

holds.

(ii) When $q<s$, by Fubini's theorem and the Minkowski inequality, we get

$$
\begin{aligned}
\left\|T_{\Omega, \alpha} f_{2}\right\|_{L^{p}(B)} & \leq\left(\int_{B}\left|\int_{2 r}^{\infty} \int_{B(0, t)}\right| f(y)|| \Omega(x-y)\left|d y \frac{d t}{t^{n+1-\alpha}}\right|^{q} d x\right)^{\frac{1}{q}} \\
& \leq \int_{2 r}^{\infty} \int_{B(0, t)}|f(y)|\left(\int_{B}|\Omega(x-y)|^{q} d x\right)^{\frac{1}{q}} d y \frac{d t}{t^{n+1-\alpha}} \\
& \leq C \int_{2 r}^{\infty} \int_{B(0, t)}|f(y)|\left(\int_{B(0, t)}|\Omega(x-y)|^{s} d x\right)^{\frac{1}{s}}|B|^{\frac{1}{q}-\frac{1}{s}} d y \frac{d t}{t^{n+1-\alpha}}
\end{aligned}
$$




$$
\begin{aligned}
& \leq C r^{\frac{n}{q}-\frac{n}{s}} \int_{2 r}^{\infty} \int_{B(0, t)}|f(y)| d y \frac{d t}{t^{n-\frac{n}{s}+1-\alpha}} \\
& \leq C r^{\frac{n}{q}-\frac{n}{s}} \int_{2 r}^{\infty}\|f\|_{L^{p}(B(0, t))} \frac{d t}{t^{\frac{n}{q}-\frac{n}{s}+1}} .
\end{aligned}
$$

Similarly, combining the above estimates, we finish this proof.

Theorem 2.5 Let $0<\alpha<n, 1<p<\frac{n}{\alpha}, \frac{1}{q}=\frac{1}{p}-\frac{\alpha}{n}$ and $\Omega \in L^{s}\left(S^{n-1}\right)$. Let $T_{\Omega, \alpha}$ be a sublinear operator $T_{\Omega}$ satisfying (1.2) and bounded from $L^{p}\left(\mathbb{R}^{n}\right)$ to $L^{q}\left(\mathbb{R}^{n}\right)$. If either of the two conditions

(i) when $s^{\prime} \leq p,\left(\varphi_{1}, \varphi_{2}\right)$ satisfies the condition

$$
\int_{r}^{\infty} \frac{\operatorname{essinf}_{t<\tau<\infty} \varphi_{1}(\tau) \tau^{\frac{n}{p}}}{t^{\frac{n}{q}+1}} d t \leq C \varphi_{2}(r)
$$

(ii) when $q<s,\left(\varphi_{1}, \varphi_{2}\right)$ satisfies the condition

$$
\int_{r}^{\infty} \frac{\operatorname{essinf}_{t<\tau<\infty} \varphi_{1}(\tau) \tau^{\frac{n}{p}}}{t^{\frac{n}{q}-\frac{n}{s}+1}} d t \leq C \varphi_{2}(r) r^{\frac{n}{s}}
$$

is satisfied, then the operator $T_{\Omega, \alpha}$ is bounded from $\dot{B}^{p, \varphi_{1}}$ to $\dot{B}^{q, \varphi_{2}}$.

Proof When $s^{\prime} \leq p$, by Lemma 2.1 and Theorem E, for $1<p<\frac{n}{\alpha}$ and $\frac{1}{q}=\frac{1}{p}-\frac{\alpha}{n}$, we have

$$
\begin{aligned}
\left\|T_{\Omega, \alpha} f\right\|_{\dot{B}^{q, \varphi_{2}}} & \leq C \sup _{r>0} \varphi_{2}(r)^{-1} \int_{r}^{\infty}\|f\|_{L^{p}(B(0, t))} \frac{d t}{t^{\frac{n}{q}+1}} \\
& =C \sup _{r>0} \varphi_{2}(r)^{-1} \int_{0}^{r^{-\frac{n}{q}}}\|f\|_{L^{p}\left(B\left(0, t^{-\frac{q}{n}}\right)\right)} d t \\
& =C \sup _{r>0} \varphi_{2}\left(r^{-\frac{q}{n}}\right)^{-1} \int_{0}^{r}\|f\|_{L^{p}\left(B\left(0, t^{-\frac{q}{n}}\right)\right)} d t \\
& \leq C \sup _{r>0} \varphi_{1}\left(r^{-\frac{q}{n}}\right)^{-1} r^{\frac{p}{q}}\|f\|_{L^{p}\left(B\left(0, r^{-\frac{q}{n}}\right)\right)}=C\|f\|_{\dot{B}^{p, \varphi_{1}}} .
\end{aligned}
$$

For the case of $q<s$, we can also use the same method to prove the desirable conclusion.

$f \in L_{\text {loc }}^{1}$, the rough fractional maximal function $M_{\Omega, \alpha}$ and the rough fractional integral $I_{\Omega, \alpha}$ are defined by

$$
\begin{aligned}
& M_{\Omega, \alpha} f(x)=\sup _{t>0} \frac{1}{|B(x, t)|^{1+\alpha}} \int_{B(x, t)}|\Omega(y)||f(y)| d y, \\
& I_{\Omega, \alpha} f(x)=\sup _{t>0} \int_{\mathbb{R}^{n}} \frac{\Omega(y) f(y)}{|x-y|^{n-\alpha}} d y
\end{aligned}
$$

for $0<\alpha<n$.

Corollary 2.6 Let $0<\alpha<n, 1<p<\frac{n}{\alpha}, \frac{1}{q}=\frac{1}{p}-\frac{\alpha}{n}$ and $\Omega \in L^{s}\left(S^{n-1}\right)$. If either of the two conditions 
(i) when $s^{\prime} \leq p,\left(\varphi_{1}, \varphi_{2}\right)$ satisfies the condition

$$
\int_{r}^{\infty} \frac{\operatorname{essinf}_{t<\tau<\infty} \varphi_{1}(\tau) \tau^{\frac{n}{p}}}{t^{\frac{n}{q}+1}} d t \leq C \varphi_{2}(r)
$$

(ii) when $q<s,\left(\varphi_{1}, \varphi_{2}\right)$ satisfies the condition

$$
\int_{r}^{\infty} \frac{\operatorname{essinf}_{t<\tau<\infty} \varphi_{1}(\tau) \tau^{\frac{n}{p}}}{t^{\frac{n}{q}-\frac{n}{s}+1}} d t \leq C \varphi_{2}(r) r^{\frac{n}{s}}
$$

is satisfied, then $M_{\Omega, \alpha}$ and $I_{\Omega, \alpha}$ are both bounded from $\dot{B}^{p, \varphi_{1}}$ to $\dot{B}^{q, \varphi_{2}}$.

Remark 1 When $s=\infty$, the comments in Theorem 2.2 and in Theorem 2.5 can be obtained from Lemmas 4.4 and 5.3 in [9].

\section{The commutators of a linear operator with rough kernel}

Let $\tilde{T}$ be a Calderón-Zygmund singular integral operator and $b \in B M O\left(\mathbb{R}^{n}\right)$. The commutator operator $[\tilde{T}, b]$ is defined by

$$
[\tilde{T}, b] f(x)=b(x) \tilde{T} f(x)-\tilde{T}(b f)(x)
$$

A well-known result of Coifman et al. [24] states that the commutator $[\tilde{T}, b]$ is bounded on $L^{p}\left(\mathbb{R}^{n}\right)$ for $1<p<\infty$ if and only if $b \in B M O\left(\mathbb{R}^{n}\right)$.

Since $B M O \subset \bigcap_{p>1} C B M O^{p, \lambda}$ when $\lambda=0$, if we only assume $b \in C B M O^{p, \lambda}$, then $[\tilde{T}, b]$ may not be a bounded operator on $L^{p}\left(\mathbb{R}^{n}\right)$. However, it has some boundedness properties on other spaces. As a matter of fact, in [25] and [26], they considered the commutators with $b \in C B M O^{p, \lambda}$. Here we also obtain some boundedness of the commutators with $b \in$ $C B M O^{p, \lambda}$ on generalized central Morrey spaces.

We need the following statement on the boundedness of the Hardy-type operator

$$
H_{1} g(t):=\frac{1}{t} \int_{0}^{t} \ln \left(1+\frac{t}{r}\right) g(r) d r, \quad 0<t<\infty
$$

Theorem F [27] The inequality

$$
\text { ess } \sup _{t>0} v_{2}(t) H_{1} g(t) \leq c \text { ess } \sup _{t>0} v_{1}(t) g(t)
$$

holds for all non-negative and non-increasing $g$ on $(0, \infty)$ if and only if

$$
A:=\sup _{t>0} \frac{\nu_{2}(t)}{t} \int_{0}^{t} \ln \left(1+\frac{t}{r}\right) \frac{d r}{\operatorname{ess} \sup _{0<\tau<r} \nu_{1}(\tau)}<\infty
$$

and $c \approx A$.

Lemma 3.1 Let $1<p<\infty, b \in C B M O^{p_{2}, \lambda}, 0<\lambda<\frac{1}{n}$ and $\frac{1}{p}=\frac{1}{p_{1}}+\frac{1}{p_{2}}$, $T_{\Omega}$ is a sublinear operator and satisfies (1.1) with $\Omega \in L^{s}\left(S^{n-1}\right)$. 
Fan Journal of Inequalities and Applications 2013, 2013:411

Page 11 of 20

When $s^{\prime} \leq p$ and $T_{\Omega}$ is bounded on $L^{p}\left(\mathbb{R}^{n}\right)$ for $1<p<\infty$, then the inequality

$$
\left\|\left[T_{\Omega}, b\right] f\right\|_{L^{p}(B(0, r))} \leq C r^{\frac{n}{p}} \int_{2 r}^{\infty} t^{n \lambda}\left(1+\ln \frac{t}{r}\right)\|f\|_{L^{p_{1}(B(0, t))}} \frac{d t}{t^{\frac{n}{p_{1}}+1}}
$$

holds for any ball $B(0, r)$ and for all $f \in L_{\text {lo }}^{p_{1}}\left(\mathbb{R}^{n}\right)$; or $p_{1}<s$ and $T_{\Omega}$ is bounded on $L^{p}\left(\mathbb{R}^{n}\right)$ for $1<p<\infty$, then the inequality

$$
\left\|\left[T_{\Omega}, b\right] f\right\|_{L^{p}(B(0, r))} \leq C r^{\frac{n}{p}-\frac{n}{s}} \int_{2 r}^{\infty} t^{n \lambda}\left(1+\ln \frac{t}{r}\right)\|f\|_{L^{p_{1}(B(0, t))}} \frac{d t}{t^{\frac{n}{p_{1}}-\frac{n}{s}+1}}
$$

holds for any ball $B(0, r)$ and for all $f \in L_{\mathrm{loc}}^{p_{1}}\left(\mathbb{R}^{n}\right)$.

Proof Let $1<p<\infty, b \in C B M O^{p_{2}, \lambda}$ and $\frac{1}{p}=\frac{1}{p_{1}}+\frac{1}{p_{2}}$. For any $r>0$, set $B=B(0, r)$ and $2 B=B(0,2 r)$. We can write

$$
f(x)=f(x) \chi_{2 B}(x)+f(x) \chi_{(2 B)^{c}}(x):=f_{1}(x)+f_{2}(x)
$$

and

$$
\begin{aligned}
{\left[T_{\Omega}, b\right] f(x)=} & \left(b(x)-b_{B}\right) T_{\Omega} f_{1}(x)-T_{\Omega}\left(\left(b(\cdot)-b_{B}\right) f_{1}\right)(x) \\
& +\left(b(x)-b_{B}\right) T_{\Omega} f_{2}(x)-T_{\Omega}\left(\left(b(\cdot)-b_{B}\right) f_{2}\right)(x) \\
= & : I_{1}+I_{2}+I_{3}+I_{4} .
\end{aligned}
$$

Hence, we have

$$
\left\|\left[T_{\Omega}, b\right] f\right\|_{L^{p}(B)} \leq\left\|I_{1}\right\|_{L^{p}(B)}+\left\|I_{2}\right\|_{L^{p}(B)}+\left\|I_{3}\right\|_{L^{p}(B)}+\left\|I_{4}\right\|_{L^{p}(B)} .
$$

Since $T_{\Omega}$ is bounded on $L^{p}\left(\mathbb{R}^{n}\right)$, it follows that

$$
\begin{aligned}
\left\|I_{1}\right\|_{L^{p}(B)} & =\left(\int_{B}\left|b(x)-b_{B}\right|^{p}\left|T_{\Omega} f_{1}(x)\right|^{p} d x\right)^{\frac{1}{p}} \\
& \leq\left(\int_{B}\left|b(x)-b_{B}\right|^{p_{2}} d x\right)^{\frac{1}{p_{2}}}\left(\int_{B}\left|T_{\Omega} f_{1}(x)\right|^{p_{1}} d x\right)^{\frac{1}{p_{1}}} \\
& \leq C r^{\frac{n}{p_{2}}+n \lambda}\|b\|_{C B M O^{p_{2}, \lambda}}\|f\|_{L^{p_{1}(2 B)}},
\end{aligned}
$$

where the constant $C>0$ is independent of $f$.

For $I_{2}$, we have

$$
\begin{aligned}
\left\|I_{2}\right\|_{L^{p}(B)} & =\left(\int_{B}\left|T_{\Omega}\left(\left(b(\cdot)-b_{B}\right) f_{1}\right)(x)\right|^{p} d x\right)^{\frac{1}{p}} \\
& \leq C\left(\int_{\mathbb{R}^{n}}\left|b(x)-b_{B}\right|^{p}\left|f_{1}(x)\right|^{p} d x\right)^{\frac{1}{p}} \\
& \leq C\left(\int_{B}\left|b(x)-b_{B}\right|^{p_{2}} d x\right)^{\frac{1}{p_{2}}}\left(\int_{B}|f(x)|^{p_{1}} d x\right)^{\frac{1}{p_{1}}} \\
& \leq C r^{\frac{n}{p_{2}}+n \lambda}\|b\|_{C B M O^{p_{2}, \lambda}}\|f\|_{L^{p_{1}(2 B)} .}
\end{aligned}
$$


Fan Journal of Inequalities and Applications 2013, 2013:411

Page 12 of 20

http://www.journalofinequalitiesandapplications.com/content/2013/1/411

For $I_{3}$, it is known that $x \in B, y \in(2 B)^{c}$, which implies $\frac{1}{2}|y| \leq|x-y| \leq \frac{3}{2}|y|$.

(i) When $s^{\prime} \leq p$ and by Fubini's theorem, we have

$$
\begin{aligned}
\left|T_{\Omega} f_{2}(x)\right| & \leq C \int_{(2 B)^{c}}|f(y)||\Omega(x-y)| \frac{d y}{|y|^{n}} \\
& =C \int_{(2 B)^{c}}|f(y)||\Omega(x-y)| \int_{|y|}^{\infty} \frac{d t}{t^{n+1}} d y \\
& =C \int_{2 r}^{\infty} \int_{2 r \leq|y|<t}|f(y)||\Omega(x-y)| d y \frac{d t}{t^{n+1}} \\
& \leq C \int_{2 r}^{\infty}\|f\|_{L^{p_{1}(B(0, t))}}\left(\int_{B(0, t)}|\Omega(x-y)|^{s} d y\right)^{\frac{1}{s}}|B(0, t)|^{1-\frac{1}{p_{1}}-\frac{1}{s}} \frac{d t}{t^{n+1}} \\
& \leq C \int_{2 r}^{\infty}\|f\|_{L^{p_{1}(B(0, t))}} \frac{d t}{t^{\frac{n}{p_{1}}+1}},
\end{aligned}
$$

thus

$$
\begin{aligned}
\left\|I_{3}\right\|_{L^{p}(B)} & \leq C\left(\int_{B}\left|b(x)-b_{B}\right|^{p} d x\right)^{\frac{1}{p}} \int_{2 r}^{\infty}\|f\|_{L^{p_{1}}(B(0, t))} \frac{d t}{t^{\frac{n}{p_{1}}+1}} \\
& \leq C r^{\frac{n}{p}+n \lambda} \int_{2 r}^{\infty}\|f\|_{L^{p_{1}}(B(0, t))} \frac{d t}{t^{\frac{n}{p_{1}}+1}} \\
& \leq C r^{\frac{n}{p}} \int_{2 r}^{\infty} t^{n \lambda}\left(1+\ln \frac{t}{r}\right)\|f\|_{L^{p_{1}(B(0, t))}} \frac{d t}{t^{\frac{n}{p_{1}}+1}} .
\end{aligned}
$$

(ii) When $p_{1}<s$, by Fubini's theorem and the Minkowski inequality, we get

$$
\begin{aligned}
& \left\|I_{3}\right\|_{L^{p}(B)} \leq\left(\int_{B}\left|\int_{2 r}^{\infty} \int_{B(0, t)}\right| b(x)-b_{B}|| f(y)|| \Omega(x-y)\left|d y \frac{d t}{t^{n+1}}\right|^{p} d x\right)^{\frac{1}{p}} \\
& \leq \int_{2 r}^{\infty} \int_{B(0, t)}|f(y)|\left(\int_{B}\left|b(x)-b_{B}\right|^{p}|\Omega(x-y)|^{p} d x\right)^{\frac{1}{p}} d y \frac{d t}{t^{n+1}} \\
& \leq \int_{2 r}^{\infty} \int_{B(0, t)}|f(y)|\left(\int_{B}\left|b(x)-b_{B}\right|^{p_{2}} d x\right)^{\frac{1}{p_{2}}}\left(\int_{B}|\Omega(x-y)|^{p_{1}} d x\right)^{\frac{1}{p_{1}}} d y \frac{d t}{t^{n+1}} \\
& \leq C r^{\frac{n}{p_{2}}+n \lambda} \int_{2 r}^{\infty} \int_{B(0, t)}|f(y)|\left(\int_{B(0, t)}|\Omega(x-y)|^{s} d x\right)^{\frac{1}{s}}|B|^{\frac{1}{p_{1}}-\frac{1}{s}} d y \frac{d t}{t^{n+1}} \\
& \leq \operatorname{Cr}^{\frac{n}{\bar{p}}-\frac{n}{s}+n \lambda} \int_{2 r}^{\infty} \int_{B(0, t)}|f(y)| d y \frac{d t}{t^{n-\frac{n}{s}+1}} \\
& \leq C r^{\frac{n}{p}-\frac{n}{s}} \int_{2 r}^{\infty} t^{n \lambda}\left(1+\ln \frac{t}{r}\right)\|f\|_{L^{p_{1}}(B(0, t))} \frac{d t}{t^{\frac{n}{p_{1}}-\frac{n}{s}+1}} .
\end{aligned}
$$

On the other hand, for $I_{4}$, by Fubini's theorem, we have

$$
\begin{aligned}
\left|\left[T_{\Omega}, b\right] f_{2}(x)\right| & \leq C \int_{(2 B)^{c}}\left|b(y)-b_{B}\right||f(y)||\Omega(x-y)| \frac{d y}{|y|^{n}} \\
& \leq C \int_{2 r}^{\infty} \int_{B(0, t)}\left|b(y)-b_{B}\right||f(y)||\Omega(x-y)| d y \frac{d t}{t^{n+1}} \\
& \leq C \int_{2 r}^{\infty} \int_{B(0, t)}\left|b(y)-b_{B(0, t)}\right||f(y)||\Omega(x-y)| d y \frac{d t}{t^{n+1}}
\end{aligned}
$$


Fan Journal of Inequalities and Applications 2013, 2013:411

Page 13 of 20

http://www.journalofinequalitiesandapplications.com/content/2013/1/411

$$
\begin{aligned}
& +C \int_{2 r}^{\infty} \int_{B(0, t)}\left|b_{B(0, r)}-b_{B(0, t)}\right||f(y)||\Omega(x-y)| d y \frac{d t}{t^{n+1}} \\
= & =I_{41}+I_{42} .
\end{aligned}
$$

(i) When $s^{\prime} \leq p$, we obtain

$$
\begin{aligned}
I_{41} \leq & C \int_{2 r}^{\infty}\left(\int_{B(0, t)}\left|b(y)-b_{B(0, t)}\right|^{p}|f(y)|^{p} d y\right)^{\frac{1}{p}} \\
& \cdot\left(\int_{B(0, t)}|\Omega(x-y)|^{s} d y\right)^{\frac{1}{s}}|B(0, t)|^{1-\frac{1}{p}-\frac{1}{s}} \frac{d t}{t^{n+1}} \\
\leq & C \int_{2 r}^{\infty}\left(\int_{B(0, t)}\left|b(y)-b_{B(0, t)}\right|^{p_{2}} d y\right)^{\frac{1}{p_{2}}}\|f\|_{L^{p_{1}(B(0, t))}} \frac{d t}{t^{\frac{n}{p}+1}} \\
\leq & C \int_{2 r}^{\infty} t^{n \lambda}\|f\|_{L^{p_{1}(B(0, t))}} \frac{d t}{t^{\frac{n}{p_{1}}+1}},
\end{aligned}
$$

then

$$
\left\|I_{41}\right\|_{L^{p}(B)} \leq C r^{\frac{n}{p}} \int_{2 r}^{\infty} t^{n \lambda}\|f\|_{L^{p_{1}(B(0, t))}} \frac{d t}{t^{\frac{n}{p_{1}}+1}} .
$$

Moreover,

$$
\begin{aligned}
I_{42} \leq & C \int_{2 r}^{\infty}\left|b_{B(0, r)}-b_{B(0, t)}\right| \int_{B(0, t)}|f(y)||\Omega(x-y)| d y \frac{d t}{t^{n+1}} \\
\leq & C \int_{2 r}^{\infty}\left|b_{B(0, r)}-b_{B(0, t)}\right|\left(\int_{B(0, t)}|f(y)|^{p_{1}} d y\right)^{\frac{1}{p_{1}}} \\
& \cdot\left(\int_{B(0, t)}|\Omega(x-y)|^{s} d y\right)^{\frac{1}{s}}|B(0, t)|^{1-\frac{1}{p_{1}}-\frac{1}{s}} \frac{d t}{t^{n+1}} \\
\leq & C \int_{2 r}^{\infty}\left(1+\ln \frac{t}{r}\right) t^{n \lambda} \mid f \|_{L^{p_{1}(B(0, t))}} \frac{d t}{t^{\frac{n}{p_{1}}+1}},
\end{aligned}
$$

then

$$
\left\|I_{42}\right\|_{L^{p}(B)} \leq C r^{\frac{n}{p}} \int_{2 r}^{\infty} t^{n \lambda}\left(1+\ln \frac{t}{r}\right)\|f\|_{L^{p_{1}(B(0, t))}} \frac{d t}{t^{\frac{n}{p_{1}}+1}} .
$$

By estimating $I_{41}$ and $I_{42}$, we obtain

$$
\left\|I_{4}\right\|_{L^{p}(B)} \leq C r^{\frac{n}{p}} \int_{2 r}^{\infty} t^{n \lambda}\left(1+\ln \frac{t}{r}\right)\|f\|_{L^{p_{1}}(B(0, t))} \frac{d t}{t^{\frac{n}{p_{1}}+1}} .
$$

(ii) When $p_{1}<s$, by the Minkowski inequality, we get

$$
\begin{aligned}
\left\|I_{41}\right\|_{L^{p}(B)} & \leq C \int_{2 r}^{\infty} \int_{B(0, t)}\left|b(y)-b_{B(0, t)}\right||f(y)|\left(\int_{B}|\Omega(x-y)|^{p} d x\right)^{\frac{1}{p}} d y \frac{d t}{t^{n+1}} \\
& \leq C r^{\frac{n}{p}-\frac{n}{s}} \int_{2 r}^{\infty} \int_{B(0, t)}\left|b(y)-b_{B(0, t)}\right||f(y)| d y \frac{d t}{t^{n-\frac{n}{s}+1}}
\end{aligned}
$$




$$
\begin{aligned}
& \leq \operatorname{Cr}^{\frac{n}{p}-\frac{n}{s}} \int_{2 r}^{\infty}\left(\int_{B(0, t)}\left|b(y)-b_{B(0, t)}\right|^{p_{2}} d y\right)^{\frac{1}{p_{2}}}\|f\|_{L^{p_{1}(B(0, t))}} t^{n-\frac{n}{p}} \frac{d t}{t^{n-\frac{n}{s}+1}} \\
& \leq C r^{\frac{n}{p}-\frac{n}{s}} \int_{2 r}^{\infty} t^{n \lambda}\|f\|_{L^{p_{1}(B(0, t))}} \frac{d t}{t^{\frac{n}{p_{1}}-\frac{n}{s}+1}}
\end{aligned}
$$

and

$$
\begin{aligned}
\left\|I_{42}\right\|_{L^{p}(B)} & \leq C \int_{2 r}^{\infty}\left|b_{B(0, r)}-b_{B(0, t)}\right| \int_{B(0, t)}|f(y)|\left(\int_{B}|\Omega(x-y)|^{p} d x\right)^{\frac{1}{p}} d y \frac{d t}{t^{n+1}} \\
& \leq C r^{\frac{n}{p}-\frac{n}{s}} \int_{2 r}^{\infty}\left|b_{B(0, r)}-b_{B(0, t)}\right| \int_{B(0, t)}|f(y)| d y \frac{d t}{t^{n-\frac{n}{s}+1}} \\
& \leq C r^{\frac{n}{p}-\frac{n}{s}} \int_{2 r}^{\infty}\left|b_{B(0, r)}-b_{B(0, t)}\right|\|f\|_{L^{p_{1}(B(0, t))}} \frac{d t}{t^{\frac{n}{p_{1}}-\frac{n}{s}+1}} \\
& \leq C r^{\frac{n}{p}-\frac{n}{s}} \int_{2 r}^{\infty} t^{n \lambda}\left(1+\ln \frac{t}{r}\right)\|f\|_{L^{p_{1}(B(0, t))}} \frac{d t}{t^{\frac{n}{p_{1}}-\frac{n}{s}+1}} .
\end{aligned}
$$

Hence, we have

$$
\left\|I_{4}\right\|_{L^{p}(B)} \leq C r^{\frac{n}{p}-\frac{n}{s}} \int_{2 r}^{\infty} t^{n \lambda}\left(1+\ln \frac{t}{r}\right)\|f\|_{L^{p_{1}}(B(0, t))} \frac{d t}{t^{\frac{n}{p_{1}}-\frac{n}{s}+1}} .
$$

Moreover, for any $q>0$, we have

$$
\begin{aligned}
r^{\frac{n}{p_{2}}+n \lambda}\|f\|_{L^{p_{1}(2 B)}} & =C r^{\frac{n}{p_{2}}+n \lambda+\frac{n}{q}}\|f\|_{L^{p_{1}(2 B)}} \int_{2 r}^{\infty} \frac{d t}{t^{\frac{n}{q}+1}} \\
& \leq C r^{\frac{n}{p_{2}}+\frac{n}{q}} \int_{2 r}^{\infty} t^{n \lambda}\|f\|_{L^{p_{1}}(B(0, t))} \frac{d t}{t^{\frac{n}{q}+1}} \\
& \leq C r^{\frac{n}{p_{2}}+\frac{n}{q}} \int_{2 r}^{\infty} t^{n \lambda}\left(1+\ln \frac{t}{r}\right)\|f\|_{L^{p_{1}(B(0, t))}} \frac{d t}{t^{\frac{n}{q}+1}} .
\end{aligned}
$$

Now combining all the above estimates, we end the proof.

Then we have the following conclusions.

Theorem 3.2 Let $1<p<\infty, b \in C B M O^{p_{2}, \lambda}, 0<\lambda<\frac{1}{n}, \frac{1}{p}=\frac{1}{p_{1}}+\frac{1}{p_{2}}$ and $\Omega \in L^{s}\left(S^{n-1}\right)$. Let $T_{\Omega}$ be a linear operator satisfying (1.1) and bounded on $L^{p}\left(\mathbb{R}^{n}\right)$ for $p>1$. If either of the two conditions

(i) when $s^{\prime} \leq p,\left(\varphi_{1}, \varphi_{2}\right)$ satisfies the condition

$$
\int_{r}^{\infty}\left(1+\ln \frac{t}{r}\right) t^{n \lambda} \frac{\operatorname{essinf}_{t<\tau<\infty} \varphi_{1}(\tau) \tau^{\frac{n}{p_{1}}}}{t^{\frac{n}{p_{1}}+1}} d t \leq C \varphi_{2}(r),
$$

(ii) when $p_{1}<s,\left(\varphi_{1}, \varphi_{2}\right)$ satisfies the condition

$$
\int_{r}^{\infty}\left(1+\ln \frac{t}{r}\right) t^{n \lambda} \frac{\operatorname{essinf}_{t<\tau<\infty} \varphi_{1}(\tau) \tau^{\frac{n}{p_{1}}}}{t^{\frac{n}{p_{1}}-\frac{n}{s}+1}} d t \leq C \varphi_{2}(r) r^{\frac{n}{s}}
$$

is satisfied, then the operator $\left[T_{\Omega}, b\right]$ is bounded from $\dot{B}^{p_{1}, \varphi_{1}}$ to $\dot{B}^{p, \varphi_{2}}$. 
Corollary 3.3 Let $1<p<\infty, b \in C B M O^{p_{2}, \lambda}, 0<\lambda<\frac{1}{n}, \frac{1}{p}=\frac{1}{p_{1}}+\frac{1}{p_{2}}$ and $\Omega \in L^{s}\left(S^{n-1}\right)$. If either of the two conditions

(i) when $s^{\prime} \leq p,\left(\varphi_{1}, \varphi_{2}\right)$ satisfies the condition

$$
\int_{r}^{\infty}\left(1+\ln \frac{t}{r}\right) t^{n \lambda} \frac{\operatorname{essinf}_{t<\tau<\infty} \varphi_{1}(\tau) \tau^{\frac{n}{p_{1}}}}{t^{\frac{n}{p_{1}}+1}} d t \leq C \varphi_{2}(r),
$$

(ii) when $p_{1}<s,\left(\varphi_{1}, \varphi_{2}\right)$ satisfies the condition

$$
\int_{r}^{\infty}\left(1+\ln \frac{t}{r}\right) t^{n \lambda} \frac{\operatorname{essinf}_{t<\tau<\infty} \varphi_{1}(\tau) \tau^{\frac{n}{p_{1}}}}{t^{\frac{n}{p_{1}}-\frac{n}{s}+1}} d t \leq C \varphi_{2}(r) r^{\frac{n}{s}}
$$

is satisfied, then the operator $\left[\tilde{T}_{\Omega}, b\right]$ is bounded from $\dot{B}^{p_{1}, \varphi_{1}}$ to $\dot{B}^{p, \varphi_{2}}$.

About the commutator of linear operator $T_{\Omega, \alpha}$ satisfying (1.2), we get the following corresponding results.

Lemma 3.4 Let $0<\alpha<n, 1<p_{1}<\frac{n}{\alpha}, b \in C B M O^{p_{2}, \lambda}, 0<\lambda<\frac{1}{n}, p_{1}^{\prime}<p_{2}<\infty$ and let

$$
\frac{1}{p}=\frac{1}{p_{1}}+\frac{1}{p_{2}}-\frac{\alpha}{n}, \quad \frac{1}{q}=\frac{1}{p_{1}}-\frac{\alpha}{n}, \quad \frac{1}{h}=\frac{1}{p_{1}}+\frac{1}{p_{2}} .
$$

$T_{\Omega-\alpha}$ is a sublinear operator and satisfies (1.2) with $\Omega \in L^{s}\left(S^{n-1}\right)$.

When $s^{\prime} \leq h, T_{\Omega, \alpha}$ is bounded from $L^{t}\left(\mathbb{R}^{n}\right)$ to $L^{m}\left(\mathbb{R}^{n}\right)$ for any $1<t<\frac{n}{\alpha}$ and $\frac{1}{m}=\frac{1}{t}-\frac{\alpha}{n}$, then the inequality

$$
\left\|\left[T_{\Omega, \alpha}, b\right] f\right\|_{L^{p}(B(0, r))} \leq C r^{\frac{n}{p}} \int_{2 r}^{\infty} t^{n \lambda}\left(1+\ln \frac{t}{r}\right)\|f\|_{L^{p_{1}(B(0, t))}} \frac{d t}{t^{\frac{n}{q}+1}}
$$

holds for any ball $B(0, r)$ and all $f \in L_{\mathrm{loc}}^{p_{1}}\left(\mathbb{R}^{n}\right)$; or $q<s, T_{\Omega, \alpha}$ is bounded from $L^{t}\left(\mathbb{R}^{n}\right)$ to $L^{m}\left(\mathbb{R}^{n}\right)$ for any $1<t<\frac{n}{\alpha}$ and $\frac{1}{m}=\frac{1}{t}-\frac{\alpha}{n}$, then the inequality

$$
\left\|\left[T_{\Omega, \alpha}, b\right] f\right\|_{L^{p}(B(0, r))} \leq C r^{\frac{n}{p}-\frac{n}{s}} \int_{2 r}^{\infty} t^{n \lambda}\left(1+\ln \frac{t}{r}\right)\|f\|_{L^{p_{1}(B(0, t))}} \frac{d t}{t^{\frac{n}{q}-\frac{n}{s}+1}}
$$

holds for any ball $B(0, r)$ and all $f \in L_{\mathrm{loc}}^{p_{1}}\left(\mathbb{R}^{n}\right)$.

Proof Let $0<\alpha<n, 1<p_{1}<\frac{n}{\alpha}, b \in C B M O^{p_{2}, \lambda}, 0<\lambda<\frac{1}{n}$. For any $r>0$, set $B=B(0, r)$ and $2 B=B(0,2 r)$. We also write

$$
f(x)=f(x) \chi_{2 B}(x)+f(x) \chi_{(2 B)^{c}}(x):=f_{1}(x)+f_{2}(x)
$$

and

$$
\begin{aligned}
{\left[T_{\Omega, \alpha}, b\right] f(x)=} & \left(b(x)-b_{B}\right) T_{\Omega, \alpha} f_{1}(x)-T_{\Omega, \alpha}\left(\left(b(\cdot)-b_{B}\right) f_{1}\right)(x) \\
& +\left(b(x)-b_{B}\right) T_{\Omega, \alpha} f_{2}(x)-T_{\Omega, \alpha}\left(\left(b(\cdot)-b_{B}\right) f_{2}\right)(x) \\
=: & I I_{1}+I I_{2}+I I_{3}+I I_{4} .
\end{aligned}
$$


Fan Journal of Inequalities and Applications 2013, 2013:411

Page 16 of 20

Hence, we have

$$
\left\|\left[T_{\Omega, \alpha}, b\right] f\right\|_{L^{p}(B)} \leq\left\|I I_{1}\right\|_{L^{p}(B)}+\left\|I I_{2}\right\|_{L^{p}(B)}+\left\|I I_{3}\right\|_{L^{p}(B)}+\left\|I I_{4}\right\|_{L^{p}(B)} .
$$

Since $T_{\Omega, \alpha}$ is bounded from $L^{p_{1}}\left(\mathbb{R}^{n}\right)$ to $L^{q}\left(\mathbb{R}^{n}\right)$ and $\frac{1}{q}=\frac{1}{p_{1}}-\frac{\alpha}{n}$, it follows that

$$
\begin{aligned}
\left\|I I_{1}\right\|_{L^{p}(B)} & =\left(\int_{B}\left|b(x)-b_{B}\right|^{p}\left|T_{\Omega, \alpha} f_{1}(x)\right|^{p} d x\right)^{\frac{1}{p}} \\
& \leq\left(\int_{B}\left|b(x)-b_{B}\right|^{p_{2}} d x\right)^{\frac{1}{p_{2}}}\left(\int_{B}\left|T_{\Omega, \alpha} f_{1}(x)\right|^{q} d x\right)^{\frac{1}{q}} \\
& \leq C r^{\frac{n}{p_{2}}+n \lambda}\|b\|_{C B M O^{p_{2}, \lambda}}\|f\|_{L^{p_{1}}(2 B)}
\end{aligned}
$$

where the constant $C>0$ is independent of $f$.

$$
\begin{aligned}
& \text { For } I I_{2}, \frac{1}{p}=\frac{1}{h}-\frac{\alpha}{n} \text { and } \frac{1}{h}=\frac{1}{p_{1}}+\frac{1}{p_{2}} \\
& \qquad \begin{aligned}
\left\|I_{2}\right\|_{L^{p}(B)} & =\left(\int_{B}\left|T_{\Omega, \alpha}\left(\left(b(\cdot)-b_{B}\right) f_{1}\right)(x)\right|^{p} d x\right)^{\frac{1}{p}} \\
& \leq\left(\int_{\mathbb{R}^{n}}\left|b(x)-b_{B}\right|^{h}\left|f_{1}(x)\right|^{h} d x\right)^{\frac{1}{h}} \\
& \leq\left(\int_{B}\left|b(x)-b_{B}\right|^{p_{2}} d x\right)^{\frac{1}{p_{2}}}\left(\int_{B}|f(x)|^{p_{1}} d x\right)^{\frac{1}{p_{1}}} \\
& \leq C r^{\frac{n}{p_{2}}+n \lambda}\|b\|_{C B M O^{p_{2}, \lambda}}\|f\|_{L^{p_{1}(2 B)}} .
\end{aligned}
\end{aligned}
$$

For $I_{3}$,

(i) when $s^{\prime} \leq h$, by Fubini's theorem, since $x \in B$, we have

$$
\begin{aligned}
\left|T_{\Omega, \alpha} f_{2}(x)\right| & \leq C \int_{(2 B)^{c}}|f(y)||\Omega(x-y)| \frac{d y}{|y|^{n-\alpha}} \\
& =C \int_{(2 B)^{c}}|f(y)||\Omega(x-y)| \int_{|y|}^{\infty} \frac{d t}{t^{n-\alpha+1}} d y \\
& =C \int_{2 r}^{\infty} \int_{2 r \leq|y|<t}|f(y)||\Omega(x-y)| d y \frac{d t}{t^{n-\alpha+1}} \\
& \leq C \int_{2 r}^{\infty}\|f\|_{L^{p_{1}(B(0, t))}}\left(\int_{B(0, t)}|\Omega(x-y)|^{s} d y\right)^{\frac{1}{s}}|B(0, t)|^{1-\frac{1}{p_{1}}-\frac{1}{s}} \frac{d t}{t^{n-\alpha+1}} \\
& \leq C \int_{2 r}^{\infty}\|f\|_{L^{p_{1}(B(0, t))}} \frac{d t}{t^{\frac{n}{q}+1}},
\end{aligned}
$$

thus

$$
\begin{aligned}
\left\|I_{3}\right\|_{L^{p}(B)} & \leq C\left(\int_{B}\left|b(x)-b_{B}\right|^{p} d x\right)^{\frac{1}{p}} \int_{2 r}^{\infty}\|f\|_{L^{p_{1}(B(0, t))}} \frac{d t}{t^{\frac{n}{q}+1}} \\
& \leq C\left(\int_{B}\left|b(x)-b_{B}\right|^{p_{2}} d x\right)^{\frac{1}{p_{2}}} r^{\frac{n}{q}} \int_{2 r}^{\infty}\|f\|_{L^{p_{1}(B(0, t))}} \frac{d t}{t^{\frac{n}{q}+1}}
\end{aligned}
$$


Fan Journal of Inequalities and Applications 2013, 2013:411

Page 17 of 20

http://www.journalofinequalitiesandapplications.com/content/2013/1/411

$$
\begin{aligned}
& \leq C r^{\frac{n}{p}+n \lambda} \int_{2 r}^{\infty}\left(1+\ln \frac{t}{r}\right)\|f\|_{L^{p_{1}(B(0, t))}} \frac{d t}{t^{\frac{n}{q}+1}} \\
& \leq C r^{\frac{n}{p}} \int_{2 r}^{\infty} t^{n \lambda}\left(1+\ln \frac{t}{r}\right)\|f\|_{L^{p_{1}(B(0, t))}} \frac{d t}{t^{\frac{n}{q}+1}} ;
\end{aligned}
$$

(ii) when $q<s$, by Fubini's theorem and the Minkowski inequality, we get

$$
\begin{aligned}
\| I_{3} & \|_{L^{p}(B)} \\
& \leq\left(\int_{B}\left|\int_{2 r}^{\infty} \int_{B(0, t)}\right| b(x)-b_{B}|| f(y)|| \Omega(x-y)\left|d y \frac{d t}{t^{n-\alpha+1}}\right|^{p} d x\right)^{\frac{1}{p}} \\
& \leq \int_{2 r}^{\infty} \int_{B(0, t)}|f(y)|\left(\int_{B}\left|b(x)-b_{B}\right|^{p}|\Omega(x-y)|^{p} d x\right)^{\frac{1}{p}} d y \frac{d t}{t^{n-\alpha+1}} \\
& \leq \int_{2 r}^{\infty} \int_{B(0, t)}|f(y)|\left(\int_{B}\left|b(x)-b_{B}\right|^{p_{2}} d x\right)^{\frac{1}{p_{2}}}\left(\int_{B}|\Omega(x-y)|^{q} d x\right)^{\frac{1}{q}} d y \frac{d t}{t^{n-\alpha+1}} \\
& \leq C r^{\frac{n}{p_{2}}+n \lambda} \int_{2 r}^{\infty} \int_{B(0, t)}|f(y)|\left(\int_{B(0, t)}|\Omega(x-y)|^{s} d x\right)^{\frac{1}{s}}|B|^{\frac{1}{q}-\frac{1}{s}} d y \frac{d t}{t^{n-\alpha+1}} \\
& \leq C r^{\frac{n}{p}-\frac{n}{s}+n \lambda} \int_{2 r}^{\infty} \int_{B(0, t)}|f(y)| d y \frac{d t}{t^{n-\frac{n}{s}-\alpha+1}} \\
& \leq C r^{\frac{n}{p}-\frac{n}{s}} \int_{2 r}^{\infty} t^{n \lambda}\left(1+\ln \frac{t}{r}\right)\|f\|_{L^{p_{1}(B(0, t))}} \frac{d t}{t^{\frac{n}{q}-\frac{n}{s}+1}} \cdot
\end{aligned}
$$

On the other hand, for $I_{4}$, by Fubini's theorem, we have

$$
\begin{aligned}
\left|\left[T_{\Omega, \alpha}, b\right] f_{2}(x)\right| \leq & C \int_{(2 B)^{c}}\left|b(y)-b_{B}\right||f(y)||\Omega(x-y)| \frac{d y}{|y|^{n-\alpha}} \\
\leq & C \int_{2 r}^{\infty} \int_{B(0, t)}\left|b(y)-b_{B}\right||f(y)||\Omega(x-y)| d y \frac{d t}{t^{n-\alpha+1}} \\
\leq & C \int_{2 r}^{\infty} \int_{B(0, t)}\left|b(y)-b_{B(0, t)}\right||f(y)||\Omega(x-y)| d y \frac{d t}{t^{n-\alpha+1}} \\
& +C \int_{2 r}^{\infty} \int_{B(0, t)}\left|b_{B(0, r)}-b_{B(0, t)}\right| f(y)|| \Omega(x-y) \mid d y \frac{d t}{t^{n-\alpha+1}} \\
= & : I_{41}+I_{42} .
\end{aligned}
$$

(i) When $s^{\prime} \leq h$, we obtain

$$
\begin{aligned}
I_{41} \leq & C \int_{2 r}^{\infty}\left(\int_{B(0, t)}\left|b(y)-b_{B(0, t)}\right|^{h}|f(y)|^{h} d y\right)^{\frac{1}{h}} \\
& \cdot\left(\int_{B(0, t)}|\Omega(x-y)|^{s} d y\right)^{\frac{1}{s}}|B(0, t)|^{1-\frac{1}{h}-\frac{1}{s}} \frac{d t}{t^{n-\alpha+1}} \\
\leq & C \int_{2 r}^{\infty}\left(\int_{B(0, t)}\left|b(y)-b_{B(0, t)}\right|^{p_{2}} d y\right)^{\frac{1}{p_{2}}}\|f\|_{L^{p_{1}(B(0, t))}} t^{n-\frac{n}{h}} \frac{d t}{t^{n-\alpha+1}} \\
\leq & C \int_{2 r}^{\infty} t^{n \lambda}\|f\|_{L^{p_{1}(B(0, t))}} \frac{d t}{t^{\frac{n}{q}+1}},
\end{aligned}
$$


Fan Journal of Inequalities and Applications 2013, 2013:411

Page 18 of 20

then

$$
\left\|I_{41}\right\|_{L^{p}(B)} \leq C r^{\frac{n}{p}} \int_{2 r}^{\infty} t^{n \lambda}\|f\|_{L^{p_{1}(B(0, t))}} \frac{d t}{t^{\frac{n}{q}+1}} .
$$

For $I_{42}$, we have

$$
\begin{aligned}
I_{42} \leq & C \int_{2 r}^{\infty}\left|b_{B(0, r)}-b_{B(0, t)}\right| \int_{B(0, t)}|f(y)||\Omega(x-y)| d y \frac{d t}{t^{n-\alpha+1}} \\
\leq & C \int_{2 r}^{\infty}\left|b_{B(0, r)}-b_{B(0, t)}\right|\left(\int_{B(0, t)}|f(y)|^{p_{1}} d y\right)^{\frac{1}{p_{1}}} \\
& \cdot\left(\int_{B(0, t)}|\Omega(x-y)|^{s} d y\right)^{\frac{1}{s}}|B(0, t)|^{1-\frac{1}{p_{1}}-\frac{1}{s}} \frac{d t}{t^{n-\alpha+1}} \\
\leq & C \int_{2 r}^{\infty}\left(1+\ln \frac{t}{r}\right) t^{n \lambda}\|f\|_{L^{p_{1}(B(0, t))}} \frac{d t}{t^{\frac{n}{q}+1}}
\end{aligned}
$$

then

$$
\left\|I_{42}\right\|_{L^{p}(B)} \leq C r^{\frac{n}{p}} \int_{2 r}^{\infty} t^{n \lambda}\left(1+\ln \frac{t}{r}\right)\|f\|_{L^{p_{1}(B(0, t))}} \frac{d t}{t^{\frac{n}{q}+1}} .
$$

Then, by estimating $I_{41}$ and $I_{42}$, we obtain

$$
\left\|I_{4}\right\|_{L^{p}(B)} \leq C r^{\frac{n}{p}} \int_{2 r}^{\infty} t^{n \lambda}\left(1+\ln \frac{t}{r}\right)\|f\|_{L^{p_{1}(B(0, t))}} \frac{d t}{t^{\frac{n}{q}+1}} .
$$

(ii) When $q<s$, by the Minkowski inequality, we get

$$
\begin{aligned}
\left\|I_{41}\right\|_{L^{p}(B)} & \leq C \int_{2 r}^{\infty} \int_{B(0, t)}\left|b(y)-b_{B(0, t)}\right| f(y) \mid\left(\int_{B}|\Omega(x-y)|^{p} d x\right)^{\frac{1}{p}} d y \frac{d t}{t^{n-\alpha+1}} \\
& \leq C r^{\frac{n}{p}-\frac{n}{s}} \int_{2 r}^{\infty} \int_{B(0, t)}\left|b(y)-b_{B(0, t)}\right||f(y)| d y \frac{d t}{t^{n-\frac{n}{s}-\alpha+1}} \\
& \leq \operatorname{Cr}^{\frac{n}{p}-\frac{n}{s}} \int_{2 r}^{\infty}\left(\int_{B(0, t)}\left|b(y)-b_{B(0, t)}\right|^{p_{2}} d y\right)^{\frac{1}{p_{2}}}\|f\|_{L^{p_{1}(B(0, t))}} t^{n-\frac{n}{h}} \frac{d t}{t^{n-\frac{n}{s}-\alpha+1}} \\
& \leq \operatorname{Cr}^{\frac{n}{p}-\frac{n}{s}} \int_{2 r}^{\infty} t^{n \lambda}\|f\|_{L^{p_{1}(B(0, t))}} \frac{d t}{t^{\frac{n}{q}-\frac{n}{s}+1}}
\end{aligned}
$$

and

$$
\begin{aligned}
\left\|I_{42}\right\|_{L^{p}(B)} & \leq C \int_{2 r}^{\infty}\left|b_{B(0, r)}-b_{B(0, t)}\right| \int_{B(0, t)}|f(y)|\left(\int_{B}|\Omega(x-y)|^{p} d x\right)^{\frac{1}{p}} d y \frac{d t}{t^{n-\alpha+1}} \\
& \leq C r^{\frac{n}{p}-\frac{n}{s}} \int_{2 r}^{\infty}\left|b_{B(0, r)}-b_{B(0, t)}\right| \int_{B(0, t)}|f(y)| d y \frac{d t}{t^{n-\frac{n}{s}-\alpha+1}} \\
& \leq C r^{\frac{n}{p}-\frac{n}{s}} \int_{2 r}^{\infty}\left|b_{B(0, r)}-b_{B(0, t)}\right|\|f\|_{L^{p_{1}(B(0, t))}} \frac{d t}{t^{\frac{n}{q}-\frac{n}{s}+1}} \\
& \leq C r^{\frac{n}{p}-\frac{n}{s}} \int_{2 r}^{\infty} t^{n \lambda}\left(1+\ln \frac{t}{r}\right)\|f\|_{\left.L^{p_{1}(B(0, t)}\right)} \frac{d t}{t^{\frac{n}{q}-\frac{n}{s}+1}}
\end{aligned}
$$


Hence, we have

$$
\left\|I_{4}\right\|_{L^{p}(B)} \leq C r^{\frac{n}{p}-\frac{n}{s}} \int_{2 r}^{\infty} t^{n \lambda}\left(1+\ln \frac{t}{r}\right)\|f\|_{L^{p_{1}(B(0, t))}} \frac{d t}{t^{\frac{n}{q}-\frac{n}{s}+1}} .
$$

Then we end this proof.

Theorem 3.5 Let $0<\alpha<n, 1<p_{1}<\frac{n}{\alpha}, b \in C B M O^{p_{2}, \lambda}, 0<\lambda<\frac{1}{n}, p_{1}^{\prime}<p_{2}<\infty$ and

$$
\frac{1}{p}=\frac{1}{p_{1}}+\frac{1}{p_{2}}-\frac{\alpha}{n}, \quad \frac{1}{q}=\frac{1}{p_{1}}-\frac{\alpha}{n}, \quad \frac{1}{h}=\frac{1}{p_{1}}+\frac{1}{p_{2}} .
$$

Let $T_{\Omega, \alpha}$ be a linear operator satisfying (1.2) with $\Omega \in L^{s}\left(S^{n-1}\right)$, which is bounded from $L^{t}\left(\mathbb{R}^{n}\right)$ to $L^{m}\left(\mathbb{R}^{n}\right)$ for any $1<t<\frac{n}{\alpha}, \frac{1}{m}=\frac{1}{t}-\frac{\alpha}{n}$. If either of the two conditions

(i) when $s^{\prime} \leq h,\left(\varphi_{1}, \varphi_{2}\right)$ satisfies the condition

$$
\int_{r}^{\infty}\left(1+\ln \frac{t}{r}\right) t^{n \lambda} \frac{\operatorname{essinf}_{t<\tau<\infty} \varphi_{1}(\tau) \tau^{\frac{n}{p_{1}}}}{t^{\frac{n}{q}+1}} d t \leq C \varphi_{2}(r)
$$

(ii) when $q<s,\left(\varphi_{1}, \varphi_{2}\right)$ satisfies the condition

$$
\int_{r}^{\infty}\left(1+\ln \frac{t}{r}\right) t^{n \lambda} \frac{\operatorname{essinf}_{t<\tau<\infty} \varphi_{1}(\tau) \tau^{\frac{n}{p_{1}}}}{t^{\frac{n}{q}-\frac{n}{s}+1}} d t \leq C \varphi_{2}(r) r^{\frac{n}{s}}
$$

is satisfied, then the operator $\left[T_{\Omega, \alpha}, b\right]$ is bounded from $\dot{B}^{p_{1}, \varphi_{1}}$ to $\dot{B}^{p, \varphi_{2}}$.

Corollary 3.6 Let $0<\alpha<n, 1<p_{1}<\frac{n}{\alpha}, b \in C B M O^{p_{2}, \lambda}, 0<\lambda<\frac{1}{n}, p_{1}^{\prime}<p_{2}<\infty, \Omega \in L^{s}\left(S^{n-1}\right)$ and

$$
\frac{1}{p}=\frac{1}{p_{1}}+\frac{1}{p_{2}}-\frac{\alpha}{n}, \quad \frac{1}{q}=\frac{1}{p_{1}}-\frac{\alpha}{n}, \quad \frac{1}{h}=\frac{1}{p_{1}}+\frac{1}{p_{2}} .
$$

If either of the two conditions

(i) when $s^{\prime} \leq h,\left(\varphi_{1}, \varphi_{2}\right)$ satisfies the condition

$$
\int_{r}^{\infty}\left(1+\ln \frac{t}{r}\right) t^{n \lambda} \frac{\operatorname{ess}^{\inf _{t<\tau<\infty}} \varphi_{1}(\tau) \tau^{\frac{n}{p_{1}}}}{t^{\frac{n}{q}+1}} d t \leq C \varphi_{2}(r)
$$

(ii) when $q<s,\left(\varphi_{1}, \varphi_{2}\right)$ satisfies the condition

$$
\int_{r}^{\infty}\left(1+\ln \frac{t}{r}\right) t^{n \lambda} \frac{\operatorname{essinf}_{t<\tau<\infty} \varphi_{1}(\tau) \tau^{\frac{n}{p_{1}}}}{t^{\frac{n}{q}-\frac{n}{s}+1}} d t \leq C \varphi_{2}(r) r^{\frac{n}{s}}
$$

is satisfied, then the operator $\left[I_{\Omega, \alpha}, b\right]$ is bounded from $\dot{B}^{p_{1}, \varphi_{1}}$ to $\dot{B}^{p, \varphi_{2}}$.

Remark 2 In our main results, if we let $\varphi_{1}=r^{n \lambda_{1}}$ and $\varphi_{2}=r^{n \lambda_{2}}$, then by calculating we can recover some known results in [7] and [25]. 


\section{Competing interests}

The author declares that they have no competing interests.

\section{Author's contributions}

The author contributed to all the main results in this paper.

\section{Acknowledgements}

This work was supported by NSF of Zhejiang Province (No. LQ13A010005). The author thanks the referees for their valuable suggestions.

\section{Received: 24 July 2012 Accepted: 8 August 2013 Published: 27 August 2013}

\section{References}

1. Morrey, CB: On the solutions of quasi-linear elliptic partial differential equations. Trans. Am. Math. Soc. 43, 126-166 (1938)

2. Peetre, J: On the theory of $M_{p, \lambda}$. J. Funct. Anal. 4, 71-87 (1969)

3. Chiarenza, J, Frasca, M: Morrey spaces and Hardy-Littlewood maximal functions. Rend. Mat. Appl. 7, $273-279$ (1987)

4. Nakai, E: Hardy-Littlewood maximal operator, singular integral operator and Riesz potentials on generalized Morrey spaces. Math. Nachr. 166, 95-103 (1994)

5. Satorn, S: Necessary and sufficient conditions for boundedness of commutators of fractional integral operators on classical Morrey spaces. Hokkaido Math. J. 35, 683-696 (2006)

6. Cao, XN, Chen, DX: The boundedness of Toeplitz-type operators on vanishing Morrey space. Anal. Theory Appl. 27, 309-319 (2011)

7. Alvarez, J, Lakey, J, Guzmán-Partuda, M: Spaces of bounded $\lambda$-central mean oscillation, Morrey spaces, and $\lambda$-central Careson measures. Collect. Math. 51, 1-47 (2000)

8. Guliyev, VS: Boundedness of maximal operator, potential and singular operators in the generalized Morrey spaces. J. Inequal. Appl. 2009, Article ID 503948 (2009)

9. Guliyev, VS, Aliyev, SS, Karaman, T, Shukurov, PS: Boundedness of sublinear operator and commutators on generalized Morrey spaces. Integral Equ. Oper. Theory 71, 327-355 (2011)

10. Akbulut, A, Guliyev, VS, Mustafayev, R: On the boundedness of the maximal operators and singular integral operators in generalized Morrey spaces. Math. Bohem. 137, 27-43 (2012)

11. Guliyev, VS: Integral operators on function spaces on the homogeneous groups and on domains in $\mathbb{R}^{n}$. Doctor's degree dissertation, Mat. Inst. Steklov, Moscow (1994)

12. Guliyev, VS: Function Spaces, Integral Operators and Two Weighted Inequalities on Homogeneous Groups. Some Applications. Casioglu, Baku (1999)

13. Guliyev, VS: Some properties of the anisotropic Riesz-Bessel potential. Anal. Math. 26, 99-118 (2000)

14. Guliyev, VS, Mustafayev, RC: Integral operators of potential type in spaces of homogeneous type. Dokl. Ross. Akad. Nauk. Mat. 354, 730-732 (1997)

15. Guliyev, VS, Mustafayev, RC: Fractional integrals in spaces of functions defined on spaces of homogeneous type. Anal. Math. 24, 181-200 (1998)

16. Burenkov, VI, Gogatishvili, A, Guliyev, VS, Mustafayev, RC: Boundedness of the Riesz potential in local Morrey-type spaces. Potential Anal. 35, 327-355 (2011)

17. Burenkov, Vl, Guliyev, HV: Necessary and sufficient conditions for boundedness of the maximal operator in the local Morrey type spaces. Stud. Math. 163, 157-176 (2004)

18. Burenkov, VI, Guliyev, HV, Guliyev, VS: Necessary and sufficient conditions for boundedness of the fractional maximal operator in the local Morrey type spaces. J. Comput. Appl. Math. 208, 280-301 (2007)

19. Burenkov, VI, Guliyev, HV, Guliyev, VS: On boundedness of the fractional maximal operator from complementary Morrey type spaces. In: The Interaction of Analysis and Geometry. Contemp. Math., vol. 424, pp. 17-32. Am. Math. Soc., Providence (2007)

20. Burenkov, VI, Guliyev, VS: Necessary and sufficient conditions for the boundedness of the Riesz potential in local Morrey type spaces. Potential Anal. 30, 211-249 (2009)

21. Burenkov, VI, Guliyev, VS, Serbetci, A, Tararykova, TV: Necessary and sufficient conditions for the boundedness of genuine singular integral operators in local Morrey type spaces. Eurasian Math. J. 1, 32-53 (2010)

22. Mustafayev, RC: On boundedness of sublinear operators in weighted Morrey spaces. Azerb. J. Math. 2, 66-79 (2012)

23. Carro, M, Pick, L, Soria, J, Stepanov, VD: On embeddings between classical Lorentz spaces. Math. Inequal. Appl. 4, 397-428 (2001)

24. Coifman, RR, Rochberg, R, Weiss, G: Factorization theorems for Hardy spaces in several variables. Ann. Math. 103 611-635 (1976)

25. Fu, ZW, Lin, Y, Lu, SZ: $\lambda$-Central BMO estimates for commutators of singular integral operators with rough kernel. Acta Math. Sin. 24, 373-386 (2008)

26. Tao, XX, Shi, YL: Multilinear commutators of Calderón-Zygmund operator on $\lambda$-central Morrey spaces. Adv. Math. 40, 47-59 (2011)

27. Guliyev, VS: Generalized weighted Morrey spaces and higher order commutators of sublinear operators. Eurasian Math. J. 3, 33-61 (2012)

doi:10.1186/1029-242X-2013-411

Cite this article as: Fan: Boundedness of sublinear operators and their commutators on generalized central Morrey spaces. Journal of Inequalities and Applications 2013 2013:411. 